\title{
Diabetes through transitions in life; from fit to frail
}

\section{William David Strain}

Diabetes and Vascular Research Centre University of Exeter Medical School Royal Devon \& Exeter Hospital Barrack Road Exeter EX2 5AX

Corresponding Author: William David Strain, Diabetes and Vascular Research Centre University of Exeter Medical School Royal Devon \& Exeter Hospital Barrack Road Exeter EX2 5AX

Received date: February 08, 2021; Accepted date: March 19, 2021; Published date: March 22,2021

Citation: William D Strain (2021) Diabetes through transitions in life; from fit to frail J. Endo and Dis; 5(2); DOI:10.31579/2640-1045/064

Copyright: () 2021, William David Strain, This is an open access article distributed under the Creative Commons Attribution License, which permits unrestricted use, distribution, and reproduction in any medium, provided the original work is properly cited.

\begin{abstract}
The accelerated ageing that occurs in diabetes results in earlier appearance of the geriatric phenotype, including frailty. Recent guidelines have stressed the need for assessing frailty in the evaluation of older adults living with diabetes. Once evaluated and identified, however, the presence of frailty marks a significant change for the individual. Treatments that have been continued and encouraged often for many years, may be discontinued. Lifeprolonging medications such GLP-1 analogues, and SGLT-2 inhibitors are replaced with therapies designed to improve quality of life, reduce glycaemic variability and stabilise the sarcopaenia that characterises frailty, such as insulin. With this, however, comes new problems, such as the risk of hypoglycaemia, the need to for capillary glucose monitoring and sub cutaneous administration. Additionally, new diagnoses that are more common in people in diabetes, such as stroke, heart disease, dementia, falls and fractures will result in further changes, with the need for care and possibly even institutionalisation.
\end{abstract}

Keywords: diabetes; geriatric phenotype; sarcopaenia; glycaemic

This review will provide a framework to navigate the changes that occur as an individual transitions through the stages of frailty, and how best to approach the multiple challenges that will occur en route, including the approaches that may be required to actively de-prescribe drugs to improve the quality of life for those living with diabetes.

As an adult living with diabetes ages, they will go through many transitions, however, none has a greater impact on their life than becoming frail. Frailty is a dynamic and complex state of physiological decline, characterized by impaired strength, fatigue, weight loss, slowed motor processing and performance, social withdrawal, decreased balance, impaired cognition, and diminished physical functioning[1]. It is a result of increased vulnerability to stressors resulting from multisystem decline in reserve and function. A key precipitant is muscle loss (sarcopaenia), which is a recognized complication of diabetic microangiopathy, and thus poorly controlled diabetes is an important risk factor with a mechanistic link, rather than a simple shared risk association [2-4]. Frailty adds a clinical value in prognosis and decision-making because frail adults are vulnerable to many adverse outcomes such as impairment of function, infection, falls, and institutionalization. Further, frail older adults have adverse pathophysiologic or functional changes not captured fully by comorbidity and disability definitions, leading to increased mortality, even after adjusting for disease diagnoses and other factors[2].

Recent guidelines have stressed the need for assessing frailty in the evaluation of older adults living with diabetes [5]. Once evaluated, frailtyspecific individualized glycaemic targets are agreed and treatment regimen may be amended. From an individual's perspective, however, presence of frailty marks a significant change not just in their targets or medication, but the interaction with the health care providers.

\section{Complications of diabetes in the frail older adult}

Like any other person living with diabetes, the risk of micro and macrovascular events is increased in the frail population, however diabetes contributes to several other components of the frailty syndrome. Diabetes is an independent predictor of depression, functional disability, falls and fractures [6]. The latter of these presents a very interesting paradox. People with diabetes tend to have a higher BMI, which, in turn, is associated with higher bone mineral density (BMD). Thus, one would anticipate a lower fracture risk. There are competing hypotheses for the observation. It may represent impairment in microarchitecture of the bone, specifically in the loadbearing trabeculae, due to underlying microvascular disease. Alternatively, it simply be a product of the increased falls risk mediated through hypoglycaemia, sarcopaenia, neuropathy or any other component of the frailty syndrome.

Cognitive decline and dementia, however, are recognized complications of diabetes in frail older adult[7]. Indeed, the rate of cognitive decline is doubled in those with diabetes compared to those without, giving rise to both Alzheimer's type and vascular dementia[8]. Predictive factors include genetic elements, such as absence of the APO E4 allele, insulin resistance and the accompanying hyperinsulinaemia, reduced $\mathrm{C}$-peptide, previous cerebral ischaemia, chronic hyperglycaemia and intercurrent hypoglycaemia. The interplay between risk of hyperglycaemia and hypoglycaemia is complex. In those without diabetes, there is a clear association between higher average glucose and cognitive decline. In those with diabetes, however, a high average glucose is associated with a higher risk of dementia, however the risk also rises as average glucose falls into a physiological range. This is thought to be due to the increased glycaemic variability seen in those with failing alpha and beta cells, and 
resultant hypos. Indeed, in a large population-based study, the risk of dementia increased by $26 \%$ after a single major hypoglycaemic episode, and almost doubled after 3 or more episodes in a year after adjustment for potential confounders such as duration of diabetes, insulin use, HbA1c and ethnicity[9].

\section{Individualising treatment targets}

As a result of the different demands and risks, treatment targets are amended as older adults become more frail. There is growing global consensus that glycaemic targets should be relaxed in older adults. However, in the only study to date that has attempted to individualise targets for older adults, after appropriate training a group of health care professionals was asked to set individualized targets for their elderly patients. In this study, a population of aged between 70 and 97, approximately $10 \%$ of whom were very frail had an average "individualized" target set of $7.0 \%(53 \mathrm{mmol} / \mathrm{mol})$ aligned with local and national guidance for a younger population[10]. When exploring the decisions behind the targets were established, frailty did not appear to be considered[11]. Although the concept of "individualized" is well accepted, the authors concluded that health care practitioners are not adept at choosing targets without a clear guidance. The recent UK consensus statement aims to address this by recommending stratification of older adults based on their frailty, into three groups; healthy older adults, moderately frail and severely frail [5]. Once stratified, HbA1c targets are allocated according to a simple chart (table 1). This also gives guidelines for choices of medications at each stage. For the fit older adult, they should be treated the same as any other adult, with metformin as first line, and choice for second agent based on co-morbidity, including the use of GLP-1 analogues for those with atherosclerotic disease and the consideration of SGLT-2 inhibitors for those with, or at high risk of future heart failure. It is important to remember that pharmacological weight loss has not been demonstrated to impart the same benefit in older adults that it has in younger patients, indeed mortality appears to be inversely associated such that after the age of 65 there is no association between obesity and mortality, and beyond 80 years greater obesity is associated with better prognosis.

\begin{tabular}{|c|c|c|c|}
\hline Patient type & Examples of patient features & Treatment Targets & De-escalation Thresholds \\
\hline Healthy & $\begin{array}{l}\text { - } \quad \text { Few coexisting chronic illnesses } \\
\text { - Cognitive and functional status } \\
\text { intact }\end{array}$ & $58 \mathrm{mmol} / \mathrm{mol}(7.5 \%)$ & $53 \mathrm{mmol} / \mathrm{mol}(7.0 \%)$ \\
\hline $\begin{array}{l}\text { Complex } \\
\quad \text { or } \\
\text { intermediate }\end{array}$ & $\begin{array}{ll}\text { - } & \text { Multiple coexisting chronic } \\
\text { illnesses } \\
\text { - } \\
\text { >2 activities of daily living } \\
\text { impairments } \\
\text { Mild-to-moderate cognitive } \\
\text { impairment }\end{array}$ & $64 \mathrm{mmol} / \mathrm{mol}(8.0 \%)$ & $58 \mathrm{mmol} / \mathrm{mol}(7.5 \%)$ \\
\hline $\begin{array}{l}\text { Very complex } \\
\text { or } \\
\text { in poor health }\end{array}$ & $\begin{array}{ll}- & \text { Long-term condition } \\
\text { - } & \text { End-stage chronic illnesses } \\
\text { - } & \text { Moderate-to-severe cognitive } \\
\text { impairment } \\
\text { - } \\
\quad \text { dependencies }\end{array}$ & $70 \mathrm{mmol} / \mathrm{mol}(8.5 \%)$ & $64 \mathrm{mmol} / \mathrm{mol}(8.0 \%)$ \\
\hline
\end{tabular}

Table 1: An overview of the assessment of older adults living with diabetes, suggested treatment targets, and threshold to consider de-escalation of medications (based on reference 5)

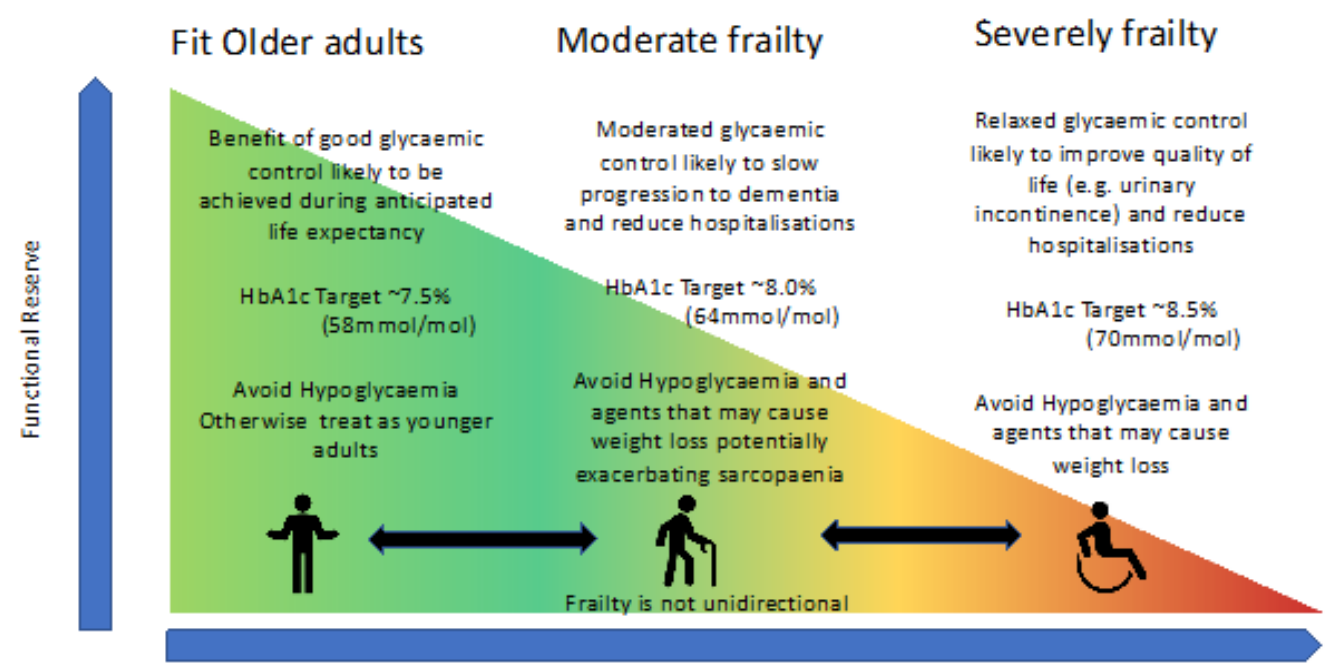

Increasing Frailty

Figure 1: Considerations for evaluating older adults. It must be remembered that frailty is potentially reversible, and should be re-evaluated after any intervention, whether that be the introduction of a new therapy or the de-escalation of a treatment that is potentially detrimental 
In the moderately frail population, the target should be set around 8.0\%, there is a role for SGLT-2 inhibitors in the setting of heart failure, and consideration should be given to GLP-1 analogues in proven atherosclerosis, particularly in the setting of stroke. However, for the patient who has not experienced an event the role of these agents may be limited given the propensity towards weight loss and the uncertainty whether this is loss of metabolically unhealthy fat or healthy muscle in the frail older adult at risk of sarcopaenia. For these patients often the weight neutral DPP-4 inhibitors can achieve target control without weight gain or loss, and have been tested in frail older adults.

For the severely frail, a target of. $8.5 \%(70 \mathrm{mmol} / \mathrm{mol})$ is established in order to minimize the risk of hypoglycaemia whilst reducing the osmotic symptoms that may lead to reduced quality of life and contribute to urinary incontinence and an increased infection risk. Given the accompanying sarcopaenia and weight loss that accompanies this degree of frailty, often these patients are best controlled with a small dose of basal insulin. The frailty induced weight loss is contributes to the phenomenon of insulin resistance burnout, characterized by the loss of insulin resistant fat tissue, and corresponding dramatic increase in insulin sensitivity. For insulin naïve patients this results in dramatic reductions in HbAlc from very modest doses of insulin. When initiating insulin in these patients, it is important to choose an insulin with the lowest risk of hypoglycaemia, such as the newer ultra-long acting analogues, as the consequences of hypoglycaemia can be devastating. The phenomenon of insulin resistance burnout, can present with significant clinical impact in people who have been taking stable doses of insulin with good glycaemic control over many years[12]. In this case, there is a rapid reduction in requirements precipitating severe hypoglycaemia without any apparent precipitant. Reducing insulin doses in these patients, however, presents a different challenge, that of reverse inertia around medication deprescribing.

\section{De-escalation of therapy}

The UK Consensus statement on the management of diabetes in frail older adults established clear guidance for de-escalation of drugs. It establishes thresholds, based on an individual's frailty for consideration of the suitability of their pharmacotherapy. These values are defined to balance the risk of hypoglycaemia and need for achieving sufficient glycaemic control to minimize complications. However, the need for de-escalation is beyond glycaemia alone. As a person becomes frail, the changes in basic physiology can result in therapies that have been well tolerated and effective for many years having different effects.

\section{Risk of polypharmacy}

Passive absorption of drugs in the stomach and ileum alter little with age, however the impact of many of many drugs on gastric motility is exaggerated in older adults. This in turn may result in increased absorption as agents spend longer passing through the lower intestine. This increased absorption is exacerbated by the reduced lean body mass and protein-binding of circulating albumin causing increased bioavailability. Additionally, reduced hepatic metabolism and glomerular filtration contribute to impaired excretion and further increased exposure. The increased exposure is compounded by the polypharmacy of older adults with multiple co-morbidities. A simple strategy of managing diabetes alone with 5 medications offers the opportunity for 10 1:1 drugdrug interactions. Include a second disease state with a further 5 medications raises the possibility of 45 interactions, a third disease with another 5 agents increases the potential to 105[13]. Although new agents are carefully evaluated for potential interactions with existing therapies within the disease they are intended for, it is very rare for potential interactions in other disease states to be tested. For example, a new drug designed to treat hyperglycaemia would be tested with existing antihyperglycaemics, and in combination with the common coprescriptions for people living with diabetes, such as statins and ACE- inhibitors. However, there is no mandate to explore potential interactions with agents used to manage different disease states, such as rheumatoid arthritis or Parkinson's disease. In practice, the risk of interactions in older adults is approximately $13 \%$ for people on 2 agents, raising to $82 \%$ at 6 medications and almost $100 \%$ once a person is on 8 medications. These interactions are responsible for approximately $10 \%$ of hospital admissions in frail older adults, up to half of which are preventable. Once an individual is on 5 or more medications, the risk of hospitalization due to adverse drug reaction rises to approximately $30 \%$ per year, one quarter of which are preventable[14].

With this overwhelming evidence of potential harm from polypharmacy, and older adults with frailty adults and diabetes being at particularly prescribed multiple agents the need for an active deprescribing policy is apparent. There are multiple steps to be taken. The first element is to determine the needs of the individual. The UK stakeholders statement provided clear guidance as to for agents to consider discontinuing as the person with diabetes transitions from healthy to moderately to severely frail. This includes considering the suitability of sulfonylureas due to the risk of hypoglycaemia and thiazolidindiones due to the fluid retention that may exacerbate heart failure and the risk of fractures. The use of GLP-1 receptor antagonists differs as patients transition into frailty, given that there is proven benefit in fit older adults by reducing the risk of atherosclerotic events whereas may exacerbate the sarcopaenia of frailty through weight loss and reduced appetite. Similarly the SgLT-2 inhibitors are probably of benefit to those with heart failure irrespective of frailty, although this has not formally been tested, but otherwise may contribute to sarcopaenia through weight loss and exacerbate the symptoms of hyperglyceamia due to increased micturition (increasing the potential for urinary incontinence) and the risk of genital infections. When considering insulin use, often twice daily biphasic insulin is used to optimize control whilst minimizing injections. However, as individuals develop frailty the need for aggressive post prandial regulation wanes, but rather simplicity takes priority. Therefore, a once daily insulin injection is often all that is required, particularly as renal function declines causing each injection to remain biologically active for longer. When external help is required to administer insulin, the use of ultra-long acting insulins such as insulin degludec or glargine u300 as they give increased flexibility for the carer who is required to attend the patient. Again, in these individuals, dosing needs to be carefully monitored, as often only a very low dose is required with renal impairment and the reduction in body fat causing a resolution of insulin resistance. There may be a temptation to discontinue insulin completely as doses are reduced, however caution should be exercised, as with long term diabetes the individual may reach a stage of insulin depletion rendering the patient prone to ketoacidosis.

\section{Deprescribing in practice}

Once a patient has transitioned into frailty and it has been decided to reevaluate their medication in order reduce the potential complications there are still multiple steps that must be accomplished. We must consider all medicines the patient is taking and identify potential risks vs benefits according to the best evidence and patient perspective and determine how these fit in with or impact on the patient's overall health goals. The most difficult element, however, is communicating these decisions to the individual. After decades of reinforcing the importance of regular medication adherence, good glycaemic control and tight blood pressure targets, to be suddenly told that medications are being discontinued can be disconcerting to a person living with diabetes. It may be perceived as rationing, cost cutting or that the health care system has given up hope for the individual and simply wishes to expedite death. Focus on the potential harm of agents and the time window to benefit, which may include candid conversations regarding the mortality of the individual. Often the compromise is that medications are held for a defined period, with an 
agreement in place for review and measures that will be taken in the event of symptoms recurring.

\section{Conclusion}

In summary one of the most significant transitions in an older adult's life is the point at which they become frail. Once recognized, the priority for treatment becomes about improving quality of life rather than prolonging it. This includes adjusting criteria, on which interventions are chosen, to tolerability and safety rather than long term risk. De-escalation of drugs also plays a major role in reducing the risk of iatrogenic complications. This, however, requires working together with the older adult living with diabetes and their families and carers in order to achieve the goal of putting life into the remaining years.

\section{Acknowledgement:}

WDS is part funded by the NIHR Exeter Clinical Research Facility. This manuscript does not necessarily reflect the views of the NIHR Exeter Clinical Research Facility, the NHS or the UK Department of Health.

\section{References}

1. Clegg, A., et al., Frailty in elderly people. The Lancet. 381(9868): p. 752-762.

2. Sinclair, A.J., A.H. Abdelhafiz, and L. Rodríguez-Mañas, Frailty and sarcopenia - newly emerging and high impact complications of diabetes. Journal of Diabetes and Its Complications. 31(9): p. 1465-1473.

3. Cruz-Jentoft, A.J. and J.P. Michel, Sarcopenia: A useful paradigm for physical frailty. European Geriatric Medicine. 4(2): p. 102105.

4. Castro-Rodríguez, M., et al., Frailty as a Major Factor in the Increased Risk of Death and Disability in Older People With Diabetes. Journal of the American Medical Directors Association. 17(10): p. 949-955.
5. Strain, W.D., et al, (2018) Type 2 diabetes mellitus in older people: a brief statement of key principles of modern day management including the assessment of frailty. A national collaborative stakeholder initiative. Diabet Med. 35(7): p. 838845.

6. Yokomoto-Umakoshi, M., et al, (2017) Association between the risk of falls and osteoporotic fractures in patients with type 2 diabetes mellitus. Endocr J. 64(7): p. 727-734.

7. Cukierman, T., H.C. Gerstein, and J.D. Williamson. (2005) Cognitive decline and dementia in diabetes--systematic overview of prospective observational studies. Diabetologia. 48(12): p. 2460-2469.

8. Cholerton, B., et al. (2016) Type 2 Diabetes, Cognition, and Dementia in Older Adults: Toward a Precision Health Approach. Diabetes Spectr. 29(4): p. 210-219.

9. Whitmer, R.A., et al. (2009) Hypoglycemic episodes and risk of dementia in older patients with type 2 diabetes mellitus. JAMA. 301(15): p. 1565-1572.

10. Strain, W.D., et al., Individualised treatment targets for elderly patients with type 2 diabetes using vildagliptin add-on or lone therapy (INTERVAL): a 24 week, randomised, double-blind, placebo-controlled study. The Lancet. 382(9890): p. 409-416.

11. Strain, W.D., A.S. Agarwal, and P.M. Paldánius. (2017) Individualizing treatment targets for elderly patients with type 2 diabetes: factors influencing clinical decision making in the 24 week, randomized INTERVAL study. Aging. 9(3): p. 769-777.

12. Abdelhafiz, A.H., L. Koay, and A.J. Sinclair. (2016) The Emergence of Frailty May Lead to a State of Burnt Out Type 2 Diabetes. J Frailty Aging. 5(3): p. 162-167.

13. Goldberg, R.M., et al. (1996) Drug-drug and drug-disease interactions in the ED: analysis of a high-risk population. Am J Emerg Med. 14(5): p. 447-450.

14. Scott, I.A., et al. (2013) Deciding when to stop: towards evidencebased deprescribing of drugs in older populations. Evid Based Med. 18(4): p. 121-124.
This work is licensed under Creative Commons Attribution 4.0 License

To Submit Your Article Click Here: Submit Manuscript

DOI: $10.31579 / 2640-1045 / 064$
Ready to submit your research? Choose Auctores and benefit from:

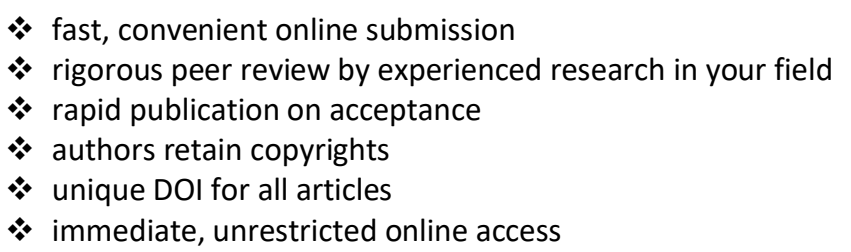

At Auctores, research is always in progress.

Learn more www.auctoresonline.org/journals/endocrinology-anddisorders 